University of Nebraska - Lincoln

DigitalCommons@University of Nebraska - Lincoln

Faculty Publications from the Department of

Electrical and Computer Engineering

Electrical \& Computer Engineering, Department

2-22-2002

\title{
Information efficiency in hyperspectral imaging systems
}

Stephen E. Reichenbach

Luyin Cao

Ram M. Narayanan

Follow this and additional works at: https://digitalcommons.unl.edu/electricalengineeringfacpub

Part of the Computer Engineering Commons, and the Electrical and Computer Engineering Commons

This Article is brought to you for free and open access by the Electrical \& Computer Engineering, Department of at DigitalCommons@University of Nebraska - Lincoln. It has been accepted for inclusion in Faculty Publications from the Department of Electrical and Computer Engineering by an authorized administrator of DigitalCommons@University of Nebraska - Lincoln. 


\title{
Information efficiency in hyperspectral imaging systems
}

\author{
Stephen E. Reichenbach \\ University of Nebraska-Lincoln \\ Computer Science and Engineering Department \\ Lincoln, Nebraska 68588 \\ E-mail: reich@unl.edu \\ Luyin Cao \\ Lattice Semiconductor Corporation \\ Colorado Springs, Colorado 80919 \\ Ram M. Narayanan \\ University of Nebraska-Lincoln \\ Electrical Engineering Department \\ Lincoln, Nebraska 68588
}

\begin{abstract}
In this work we develop a method for assessing the information density and efficiency of hyperspectral imaging systems that have spectral bands of nonuniform width. Imaging system designs with spectral bands of nonuniform width can efficiently gather information about a scene by allocating bandwidth among the bands according to their information content. The information efficiency is the ratio of information density to data density and is a function of the scene's spectral radiance, hyperspectral system design, and signal-to-noise ratio. The assessment can be used to produce an efficient system design. For example, one approach to determining the number and width of the spectral bands for an informationefficient design is to begin with a design that has a single band and then to iteratively divide a band into two bands until no further division improves the system's efficiency. Two experiments illustrate this approach, one using a simple mathematical model for the scene spectral-radiance autocorrelation function and the other using the deterministic spectral-radiance autocorrelation function of a hyperspectral image from NASA's Advanced Solid-State Array Spectroradiometer. The approach could be used either to determine a fixed system design or to dynamically control a system with variable-width spectral bands (e.g., using on-board processing in a satellite system). (c) 2002 SPIE and IS\&T.

[DOI: $10.1117 / 1.1482097]$
\end{abstract}

\section{Introduction}

In the design of imaging systems, it is typically important to deliver as much information as possible about the scene radiance with as small a data rate as possible. Hyperspectral sensors can yield large amounts of spectral-radiance data containing important information about a scene; however, in a system design, there are practical limitations on the volume of data and associated trade-offs involving spectral, spatial, and temporal coverage and resolution. This is espe-

Paper 01029 received May 11, 2001; revised manuscript received Jan. 17, 2002; accepted for publication Feb. 22, 2002.

$1017-9909 / 2002 / \$ 15.00$ @ 2002 SPIE and IS\&T. cially true in satellite remote sensing, where power and weight considerations limit the volume of data that can be communicated to receiving stations and where there are competing demands for global coverage and high spectral, spatial, and temporal resolution.

Information efficiency is the design goal of maximizing the information from an imaging system relative to the data it generates. "What constitutes information?" is a difficult question and the answer may be quite application dependent. Quantifying information is even more difficult. While it is important to recognize the limitations of any general, application-independent measure of information for specific applications, Shannon's work in information theory ${ }^{1,2}$ does provide a basis for a quantitative approach that can yield useful insights and guidance for system design. Using an information-theoretic approach, Huck and Park ${ }^{3}$ defined information efficiency as the ratio of information density to data density. This approach recognizes the distinction between data and information, e.g., additional data that is redundant or noisy may contribute little or no additional information about a scene.

One of the most important design choices for hyperspectral imaging systems is the number and width of the spectral bands. In our previous work ${ }^{4}$ we developed an approach that analyzed the information efficiency of hyperspectral imaging systems with spectral bands of uniform width. The information efficiency is computed as a function of the hyperspectral system design, signal-to-noise ratio, and autocorrelation of the scene spectral radiance. The approach allows determination of the number of uniform-width bands to optimize information efficiency for an ensemble of scenes.

The research described in this paper considers the analysis and design of hyperspectral imaging systems with spectral bands of nonuniform width. Scene radiance may 
vary statistically with respect to spectral wavelength. Designs with spectral bands of nonuniform width can allocate bands in spectral wavelengths that have more information ${ }^{5}$ so such designs can achieve greater information efficiency than designs with uniform-width spectral bands.

In Sec. 2 we present a model of the scene and imaging process. In Sec. 3 we describe computation of the information density and efficiency. Illustrated in Sec. 4 is an approach to system design with two experiments. The approach begins with a system design with a single band and iteratively divides one band in the current design as long as information efficiency improves. The first experiment uses a simple mathematical model for the scene spectralradiance autocorrelation and the second experiment uses the deterministic spectral-radiance autocorrelation of a hyperspectral image from NASA's Advanced Solid-State Array Spectroradiometer (ASAS). Section 5 concludes with a summary and discussion of the utility of the approach.

\section{Scene and Acquisition Models}

Here in Sec. 2 we describe the scene and image acquisition models that are used in analyzing the information density and efficiency. The models are presented for both the spectral-radiance domain and the Fourier transform domain.

\subsection{Nonhomogeneous Spectral Statistics of the Scene Radiance}

Sources of variability in the scene radiance include the illumination source and geometry, viewing geometry, atmospheric conditions, and the composition of the scene. ${ }^{6}$ Let the scene spectral-radiance available for acquisition be $s(\lambda)$, where $\lambda$ denotes the wavelength. Next, consider an ensemble of spectral-radiance values (e.g., over a range of time and space). We can statistically characterize the expected scene spectral radiance if we have enough independent samples. The scene radiance may not be statistically homogeneous with respect to wavelength, i.e., the statistical expectations for the scene's radiance may be wavelength dependent.

The generalized nonhomogeneous scene spectralradiance autocorrelation function for the ensemble is

$\Phi_{s}(\Delta \lambda ; \lambda)=\left\langle s\left(\lambda-\frac{\Delta \lambda}{2}\right) s\left(\lambda+\frac{\Delta \lambda}{2}\right)\right\rangle, \quad \lambda_{0} \leqslant \lambda<\lambda_{M}$

where $\langle\ldots\rangle$ denotes the expected or ensemble average of the quantity within the angular brackets, $\Delta \lambda$ denotes the interval between two wavelengths located symmetrically about wavelength $\lambda$, and the spectral range of scene radiance $s$ is taken to be $\lambda_{0}, \ldots, \lambda_{M}$. For notational convenience, the scene spectral radiance $s(\lambda)$ is taken to be a zero-mean process at all wavelengths. In practice, it is a simple matter to account for the mean in any processing.

Let $\hat{s}(\omega)$ be the Fourier transform of $s(\lambda)$ :

$\hat{s}(\omega)=\int s(\lambda) \exp (-i \omega \lambda) d \lambda$.
Following the work of Bendat and Piersol, ${ }^{7}$ the instantaneous Fourier transform for the autocorrelation function at wavelength $\lambda$ is

$\hat{\Phi}_{s}(\omega ; \lambda)=\int \Phi_{s}(\Delta \lambda ; \lambda) \exp (-i \omega \Delta \lambda) d(\Delta \lambda)$.

\subsection{Hyperspectral Image Acquisition Model}

The hyperspectral imaging system converts the scene radiance $s(\lambda)$ into a discrete set of values, one for each band. This process is modeled in three parts. First, the scene radiance over the range of each band is integrated. Second, the integration is sampled to a discrete value. Third, noise is inevitably introduced into the acquisition process.

\subsubsection{Spectral response}

A hyperspectral imaging system integrates the scene radiance in multiple bands. For each band, there is a spectral response over a range of wavelengths. Let $M$ denote the number of spectral bands. Then, the response in band $m$, $m \in\{1, \ldots, M\}$ is the integrated product of scene radiance $s(\lambda)$ and the spectral response function for that band $h_{m}(\lambda)$. Mathematically, the integrated product is the convolution of the scene spectral-radiance function with a band-dependent response function:

$r(\lambda)=\int s(\Delta \lambda) h_{m}(\lambda-\Delta \lambda) d(\Delta \lambda), \quad \lambda_{m-1} \leqslant \lambda<\lambda_{m}$,

where $\frac{1}{2}\left(\lambda_{m-1}+\lambda_{m}\right)$ is the midpoint of band $m$.

The expression for the autocorrelation function of the integrated product is complicated by the band dependence (or shift variance) of the response function. However, the autocorrelation can be approximated in a form that is more easily evaluated by assuming the principle of locality applies - that the most important aspects of the autocorrelation are in a small, central locality and that differences in the response functions of neighboring bands are small enough to be ignored. Then, the autocorrelation can be written

$$
\begin{aligned}
\Phi_{r}(\Delta \lambda ; \lambda)= & \left\langle r\left(\lambda-\frac{\Delta \lambda}{2}\right) r\left(\lambda+\frac{\Delta \lambda}{2}\right)\right\rangle \\
= & \iint \Phi_{s}(\Delta u ; u) \Phi_{h}(\Delta \lambda-\Delta u ; \lambda-u) \\
& \times d(\Delta u) d u,
\end{aligned}
$$

where

$\Phi_{h}(\Delta \lambda ; \lambda)=h_{m}\left(\lambda-\frac{\Delta \lambda}{2}\right) h_{m}\left(\lambda+\frac{\Delta \lambda}{2}\right), \quad \lambda_{m-1} \leqslant \lambda<\lambda_{m}$.

Note that even assuming the principle of locality in this expression, the autocorrelation function is band dependent.

Then, the instantaneous Fourier transform of the autocorrelation function at wavelength $\lambda$ is 
$\hat{\Phi}_{r}(\omega ; \lambda)=\int \Phi_{r}(\Delta \lambda ; \lambda) \exp (-i \omega \Delta \lambda) d(\Delta \lambda)$.

\subsubsection{Discrete bands}

The acquisition model in this research allows spectral bands of nonuniform width. There is a single value sampled for each of the $M$ bands:

$q(\lambda)=r(\lambda) \sum_{m=1}^{M} \delta\left(\lambda-\frac{1}{2}\left(\lambda_{m-1}+\lambda_{m}\right)\right)$,

where $\delta$ is the Dirac sampling impulse at the center of each band. Then, let $\Lambda_{m}$ be the width of band $m$ :

$\Lambda_{m}=\lambda_{m}-\lambda_{m-1}$.

In discrete form, the array of sampled values is

$q[m]=q\left(\lambda_{m}-\frac{\Lambda_{m}}{2}\right) \quad m \in\{1, \ldots, M\}$.

Information is lost in this sampling of a value for each band. This information loss associated with band sampling is easily seen in the Fourier domain. Assuming the principle of locality with respect to the width of the bands, the instantaneous transform contains a term of aliased transform components caused by sampling and the expected power is

$$
\begin{aligned}
\hat{\Phi}_{q}(\omega ; m)= & \hat{\Phi}_{r}\left(\omega ; \lambda_{m}-\frac{\Lambda_{m}}{2}\right) \\
& +\sum_{n \neq 0} \hat{\Phi}_{r}\left(\omega-\frac{n}{\Lambda_{m}} ; \lambda_{m}-\frac{\Lambda_{m}}{2}\right), \\
& m \in\{1, \ldots, M\} .
\end{aligned}
$$

This formulation assumes the side bands that alias to a frequency are uncorrelated. ${ }^{3}$

\subsubsection{Noise}

Inevitably, noise is present in the acquired image. Additive, signal-independent noise in the discrete values is modeled as

$p[m]=q[m]+e[m], \quad m \in\{1, \ldots, M\}$,

where $e[m]$ is the noise in band $m$. And, the instantaneous transform power is corrupted by the addition of the noise transform power:

$\hat{\Phi}_{p}(\omega ; m)=\hat{\Phi}_{q}(\omega ; m)+\hat{\Phi}_{e}(\omega ; m), \quad m \in\{1, \ldots, M\}$.

\section{Spectral Information Density and Information Efficiency}

Huck and co-workers ${ }^{3,8}$ formulated the spatial information density and efficiency to assess the design of the spatial response of a digital imaging system. In their approach, information density is a measure of the information capacity of the imaging system for an ensemble of scenes which are characterized by the autocorrelation or power spectrum. The information density is determined by the autocorrelation (or power spectrum) of the scene, the image acquisition function (or system transfer function), and the autocorrelation (or power spectrum) of the noise. The system information capacity is limited to the entropy of the image less the entropy of the noise.

Here, we formulate the spectral-radiance information density and efficiency to assess hyperspectral imaging systems. Although Huck and co-workers assumed homogeneous scene statistics (as is generally appropriate in the spatial domain), the formulation can be generalized for nonhomogeneous scene statistics (as may be expected in the spectral-radiance domain and which are described in Sec. 2.1).

From the model presented in Sec. 2, the information density of the acquired spectral signal $s(\lambda)$ in band $m$ can be derived as

$$
\begin{aligned}
H_{m}= & \frac{1}{2} \int_{\hat{B}_{m}} \\
& \times \log _{2}\left(1+\frac{\hat{\Phi}_{r}\left(\omega ; \lambda_{m}-\frac{\Lambda_{m}}{2}\right)}{\sum_{n \neq 0} \hat{\Phi}_{r}\left(\omega-\frac{n}{\Lambda_{m}} ; \lambda_{m}-\frac{\Lambda_{m}}{2}\right)+\hat{\Phi}_{e}(\omega ; m)}\right) d \omega,
\end{aligned}
$$

where $\hat{B}_{m}$ is the sampling pass band,

$\hat{B}_{m}=\left\{\omega,|\omega|<\frac{1}{2 \Lambda_{m}}\right\}$.

The unit of measure for Eq. (14) is bits of information, which Huck and co-workers termed bifs, per band. If the acquired signal $s(\lambda)$ is quantized with $\eta$-bit encoding (for $2^{\eta}$ levels), the data density in band $m$ is

$D_{m}=\frac{\eta}{\Lambda_{m}}$

The unit of measure is bits per band.

With respect to the number and spacing of spectral bands, information density $H$ increases with more, narrower spectral bandwidths. However, the data density also increases with narrower spectral bands. The trade-off between the desire for information and the cost of data can be expressed as a ratio of information efficiency, $H / D$. Considering all bands, the total information density for the system is

$H=\sum_{m=1}^{M} H_{m} \cdot \frac{\Lambda_{m}}{R}$,

where $R$ is the wavelength range (i.e., $\lambda_{M}-\lambda_{0}$ ). In a similar way, the total data density for the system is 
$D=\sum_{m=1}^{M} D_{m} \cdot \frac{\Lambda_{m}}{R}=\frac{M \eta}{R}$.

Then, the system information efficiency is

$\frac{H}{D}=\frac{1}{M \eta} \sum_{m=1}^{M} \Lambda_{m} H_{m}$

As can be seen in Eq. (14), the information density and hence the information efficiency is a function of the scene's instantaneous spectral power spectrum $\hat{\Phi}_{s}(\omega ; \lambda)$, the system spectral transfer function $\hat{h}_{m}(\omega ; \lambda)$, the spectral-band intervals $\Lambda_{m}$, and the system noise power spectrum $\hat{\Phi}_{s}(\omega ; m)$. In Sec. 4 we illustrate with two experiments how this analysis can be used to design a hyperspectral imaging system with high information efficiency.

\section{Experimental Designs}

Two experiments are now presented that illustrate the use of information efficiency in hyperspectral imaging system design. The first experiment uses a simple model for the scene radiance autocorrelation function. The second experiment uses the deterministic autocorrelation function of a hyperspectral image from the ASAS. ${ }^{9}$

The experiments illustrate an approach to determining the number and width of the spectral bands. In these experiments, the expected scene spectral radiance, system spectral response, and system noise are fixed, so the analysis considers only the problem of determining the number and width of the spectral bands for the given spectralradiance autocorrelation function. The process proceeds as follows.

1. Begin with a design that has single spectral band over the full spectral range. Analyze the information efficiency.

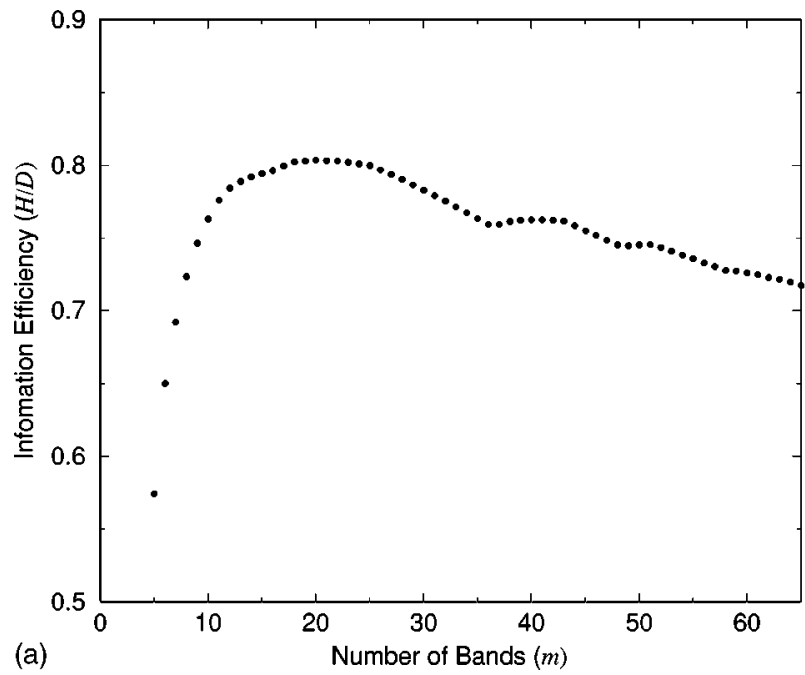

2. For each band in the current design, consider subdividing the band into two equally sized bands. Analyze the information efficiency for each such subdivision.

3. If no subdivision in step 2 increases information efficiency, then stop the process and report the current design. Otherwise, the current design is updated with the subdivision in step 2 that yielded the greatest information efficiency and steps 2 and 3 are repeated.

The result is a highly efficient design, although not necessarily optimal by the criterion of information efficiency. If a predetermined number of bands is desired, the stop criterion in step 3 can be replaced by a test of the number of bands in the current design.

\subsection{Simulation Based on a Hyperspectral Scene Model}

The first experiment assumes a simple wavelengthdependent Gaussian autocorrelation function of the scene radiance $s(\lambda)$ :

$\Phi_{s}(\Delta \lambda ; \lambda)=\sigma_{s}^{2} \exp \left(\frac{-\Delta \lambda}{\mu(\lambda)}\right)$

where $\sigma_{s}^{2}$ is the signal variance and the wavelengthdependent mean-spectral detail is a simple linear function:

$\mu(\lambda)=\frac{\mu_{M}-\mu_{0}}{\lambda_{M}-\lambda_{0}}\left(\lambda-\lambda_{0}\right)+\mu_{0}$

for $\lambda_{0} \leqslant \lambda \leqslant \lambda_{M}$. In the experiment, this simple model is parameterized over a dimensionless wavelength range between $\lambda_{0}=1$ and $\lambda_{M}=512$ and with mean-spectral detail between $\mu_{0}=50$ and $\mu_{M}=1$. With these values, the correlation between scene spectral components at smaller wavelengths is larger and the correlation between scene spectral components at larger wavelengths is smaller.

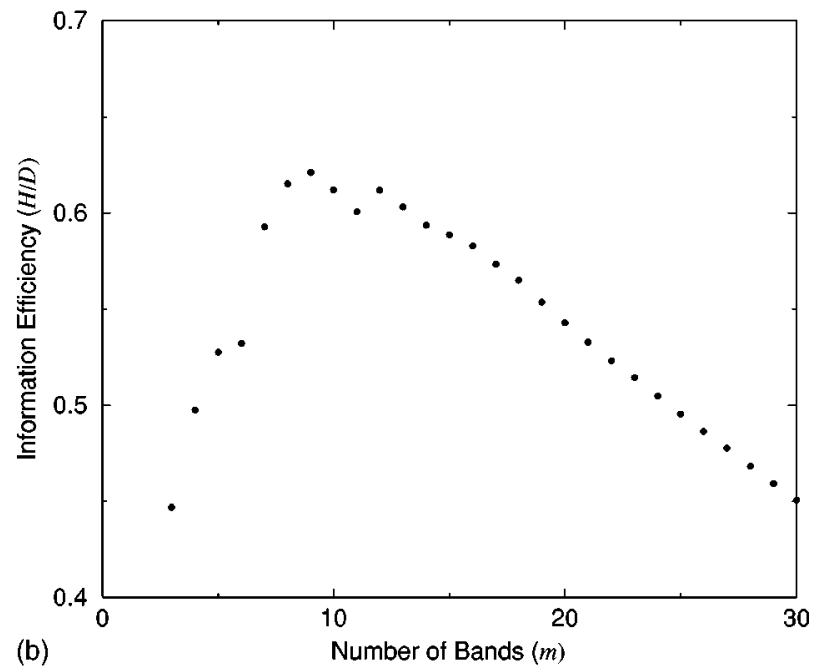

Fig. 1 Information efficiency $H / D$ as a function of the number of bands $m$. 
Table 1 Band number $m$, size $\Lambda_{m}$, and information efficiency $H_{m} / D_{m}$ of each band in the design with highest efficiency for the hyperspectral autocorrelation model in experiment 1 .

\begin{tabular}{ccccccccccccccccccccc}
\hline \hline$m$ & 1 & 2 & 3 & 4 & 5 & 6 & 7 & 8 & 9 & 10 & 11 & 12 & 13 & 14 & 15 & 16 & 17 & 18 & 19 & 20 \\
\hline$\Lambda_{m}$ & 32 & 32 & 32 & 32 & 32 & 16 & 16 & 16 & 16 & 16 & 16 & 16 & 16 & 16 & 16 & 16 & 16 & 16 & 16 & 128 \\
$H_{m} / D_{m}$ & 0.85 & 0.85 & 0.85 & 0.85 & 0.84 & 0.82 & 0.82 & 0.82 & 0.82 & 0.82 & 0.82 & 0.82 & 0.82 & 0.81 & 0.81 & 0.81 & 0.80 & 0.79 & 0.78 & 0.44 \\
\hline \hline
\end{tabular}

The system spectral response function is modeled as the convolution of a square pulse and Gaussian:

$h_{m}(\lambda)=\frac{1}{2 \Lambda_{m}} \operatorname{rect}\left(\frac{\lambda}{2 \Lambda_{m}}\right) \otimes \frac{1}{\sqrt{2 \pi \sigma_{h}^{2}}} \exp \left(-\frac{\lambda^{2}}{\sigma_{h}^{2}}\right)$.

In the experiment, the width of the Gaussian is fixed to $\sigma_{h}^{2}=1$. Although it is not considered a design variable in this experiment, the spectral response function is critical to system performance and plays a central role in the trade-off between blurring and aliasing. In this experiment, we assume signal-independent white noise (i.e., a flat noise power spectrum) with a signal-to-noise ratio of $\sigma_{s} / \sigma_{n}=8$. The experiment assumes 8-bit quantization, $\eta=8$.

Figure 1(a) illustrates the relationship between the number of bands and the information efficiency for this experiment using this approach. The information efficiency peaks at just over 0.80 with 20 bands, with $H=128.5$ bits and $D=160$ bits over the entire spectral range. The width and information efficiency of each band are presented in Table 1 .

The linearity of the scene spectral-autocorrelation parameter facilitates interpreting the reasons for the widths of the bands. At the smaller wavelengths, the autocorrelation is greater, so there is less aliasing due to band sampling and not much more information is gained by subdividing bands. At the larger frequencies, the autocorrelation is smaller, so there is significant information but the data cost of bands that are small enough to sufficiently attenuate aliasing (and so yield that information) is very high. At the middle frequencies, there is less correlation (and so more information) than at smaller frequencies and the correlation is not so small as to require very small bands (as are required at higher frequencies).

This approach yields superior results compared to the optimal design with uniform-sized spectral bands. For the same experimental design and a system design with uniform-sized bands, ${ }^{4}$ the maximum efficiency of 0.78 is achieved with 32 bands of size $\Lambda=16$. Using this approach for designing a system with nonuniform spectral bands, the information efficiency with 32 bands is 0.79 .

\subsection{Simulation Based on a Hyperspectral Scene}

The hyperspectral autocorrelation model used for the experiment presented in Sec. 4.1 is contrived as a simple-tounderstand nonhomogeneous autocorrelation function. The experiment presented here in Sec. 4.2 uses an autocorrelation function determined from a 64-band (2000 $\times 512$ pixel) image of the Washington, DC, mall from NASA's ASAS. ${ }^{9}$ The wavelength-dependent deterministic autocorrelation function is computed from this image as

$$
\begin{aligned}
& \Phi_{s}(\Delta \lambda ; \lambda) \\
& = \begin{cases}\frac{1}{N} \sum_{n} s_{n}\left(\lambda_{0}\right) s_{n}\left(\lambda_{0}+\Delta \lambda\right), & \text { if } \lambda-\frac{\Delta \lambda}{2}<\lambda_{0}, \\
\frac{1}{N} \sum_{n} s_{n}\left(\lambda-\frac{\Delta \lambda}{2}\right) s_{n}\left(\lambda+\frac{\Delta \lambda}{2}\right), & \text { otherwise, } \\
\frac{1}{N} \sum_{n} s_{n}\left(\lambda_{M}-\Delta \lambda\right) s_{n}\left(\lambda_{M}\right), & \text { if } \lambda+\frac{\Delta \lambda}{2}>\lambda_{M},\end{cases}
\end{aligned}
$$

where $s_{n}(\lambda)$ is the $n$th pixel of $N=2000 \times 512$ pixels in ASAS band $\lambda$ with $\lambda=1, \ldots, 64$. The computed autocorrelation function, shown in Fig. 2, is clearly nonhomogeneous with respect to wavelength. The autocorrelation is virtually zero in the very low bands and very high bands because there is virtually no signal in these bands of the ASAS image. There are highly correlated bands in the ASAS image centered at $\lambda=18$ and 41 and there is a narrow range of bands with lower correlation centered at $\lambda$ $=30$.

The goal of the experiment is to design nonuniform bands for this autocorrelation function to achieve high information efficiency. The system response function is identical to the model in Eq. (22) in Sec. 4.1. This experiment also assumes the same signal-independent white noise with signal-to-noise ratio $\sigma_{s} / \sigma_{n}=8$ and 8-bit quantization, $\eta$ $=8$.

Figure 1(b) illustrates the relationship between the number of bands and the information efficiency for this experiment. The information efficiency peaks at just over 0.62 with nine bands, with $H=44.7$ bits and $D=72$ bits over the entire spectral range. The width and information efficiency of each band is presented in Table 2. As in the previous experiment, the smallest bands (here, with width $\Lambda_{m}=4$ ) are in spectral regions where the autocorrelation function is not very narrow and not very broad.

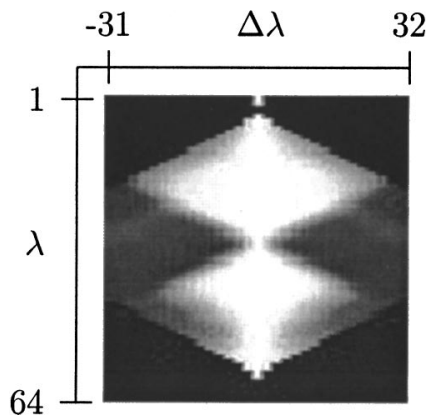

Fig. 2 Deterministic autocorrelation function of the ASAS scene. 
Table 2 Band number $m$, size $\Lambda_{m}$, and information efficiency $H_{m} / D_{m}$ of each band in the design with highest efficiency for the ASAS autocorrelation function in experiment 2.

\begin{tabular}{cccccccccc}
\hline \hline$m$ & 1 & 2 & 3 & 4 & 5 & 6 & 7 & 8 & 9 \\
\hline$\Lambda_{m}$ & 8 & 4 & 4 & 16 & 8 & 8 & 4 & 4 & 8 \\
$H_{m} / D_{m}$ & 0.58 & 0.67 & 0.55 & 0.47 & 0.82 & 0.59 & 0.61 & 0.68 & 0.61 \\
\hline \hline
\end{tabular}

This approach yields superior results compared to the optimal design with uniform-size spectral bands. For the same experimental design and a system design with uniform-size bands, ${ }^{4}$ the maximum efficiency of 0.59 is achieved with eight bands of size $\Lambda=8$. Using this approach for designing a system with nonuniform spectral bands, the information efficiency with eight bands is 0.62 .

\section{Conclusion}

The method developed in this paper assesses the information density and efficiency of hyperspectral imaging systems with spectral bands of nonuniform width. The analysis quantifies information efficiency by considering the scene statistics, system design, and signal-to-noise ratio. The assessment can be used to produce system designs that are highly efficient at gathering information. This informationtheoretic approach is application independent and does not take into account application-specific issues such as what classes of scene elements may be most important to distinguish and what wavelengths may be obscured by the environment. Of course, where a priori knowledge of the application is available, this application-independent, information-theoretic approach should be used in conjunction with knowledge of the domain.

Two experiments illustrate the approach by iteratively refining the system design for particular scene characteristics. One experiment employs a simple, easily understood mathematical model for the spectral-radiance autocorrelation function and the other experiment uses a deterministic spectral-radiance autocorrelation function of a hyperspectral image from NASA's Advanced Solid-State Array Spectroradiometer. The designs achieve greater information efficiency than optimal designs with spectral bands of uniform size.

The approach could be used either to determine a fixed system design or to dynamically control a system with variable-width spectral bands. For example, on-board processing in a satellite system could dynamically determine the spectral band design based on scene characteristics.

The approach has promise for the problem of assessing information gathering for problems of pattern recognition and classification. Rather than seeking to maximize mutual information between measured values and the actual radiance, the goal might be restated as seeking to maximize the information available to distinguish patterns. We have begun work to investigate this problem.

\section{Acknowledgments}

This research was supported by NASA and by the Nebraska Research Initiative for Geospatial Decision Support. The authors extend their appreciation to the Landsat 7 Project Science Office and the Biospheric Sciences Branch at
NASA Goddard Space Flight Center, especially to Darrel Williams, Jim Irons, John Barker, and Brian Markham.

\section{References}

1. C. Shannon, "The mathematical theory of communication," Bell Syst. Tech. J. 27, 379-423 (1948)

2. C. Shannon, "The mathematical theory of communication," Bell Syst. Tech. J. 28, 623-656 (1948).

3. F. O. Huck and S. K. Park, "Optical-mechanical line-scan imaging process: Its information capacity and efficiency," Appl. Opt. 14(10), 2508-2520 (1975).

4. S. E. Reichenbach, Y. Xiong, R. Narayanan, and L. Cao, "Information theoretic assessment and design of hyperspectral imaging systems," in Visual Information Processing, S. K. Park and R. D. Juday, eds., Proc. SPIE 3716, 100-104 (1999).

5. L. Cao, S. E. Reichenbach, and R. Narayanan, "Information theoretic assessment and design of hyperspectral imaging systems with nonuniform bandwidths," in Visual Information Processing, S. K. Park and R. D. Juday, Eds., Proc. SPIE 4388, 72-78 (2001).

6. P. F. Valdez, G. W. Donohoe, M. R. Descour, and S. Motomatsu, "Selection of spectral bands for interpretation of hyperspectral remotely sensed images," in Imaging Spectrometry II, M. R. Descour and J. M. Mooney, Eds., Proc. SPIE 2819, 195-203 (1996).

7. J. S. Bendat and A. G. Piersol, Measurement and Analysis of Random Data, Wiley, New York (1966).

8. S. John, Z-u. Rahman, F. O. Huck, and S. E. Reichenbach, "Information theoretical assessment of digital imaging systems," in Infrared Imaging Systems: Design, Analysis, Modeling, and Testing, G. C. Holst, Ed., Proc. SPIE 309, 53-66 (1990).

9. J. Irons, K. Ranson, D. Williams, R. Irish, and F. Huegel, "An offnadir pointing imaging spectroradiometer for terrestrial ecosystem studies," IEEE Trans. Geosci. Remote Sens. 29(1), 66-74 (1991).

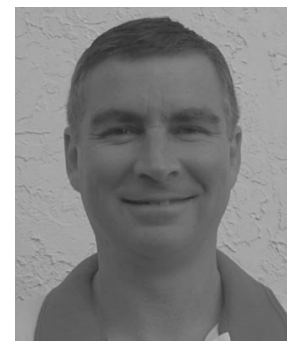

Stephen E. Reichenbach is an associate professor at the Computer Science and Engineering (CSE), Department of the University of Nebraska-Lincoln (UNL). He earned his $\mathrm{PhD}$ in computer science from the College of William and Mary, his master's degree in computer science from Washington University in St. Louis, and his bachelor's degree in English from the University of Nebraska. His postdoctoral positions include a National Research Council research associateship at the Visual Information Processing Laboratory, NASA Langley Research Center, and an ASEE research fellowship at the Landsat 7 Project Science Office, NASA Goddard Space Flight Center. From 1996 to 2000, he served as UNL CSE department chair and currently is a senior associate to the UNL vice chancellor for research. He has published more than 50 journal and conference papers on digital image processing and image information systems.

Luyin Cao is a software engineer with Lattice Semiconductor Corporation, Colorado Springs, Colorado. She earned her master's degree in computer science from the University of Nebraska-Lincoln in 2000 and holds BS and MS degrees from Anhui University (Hefei, China). 


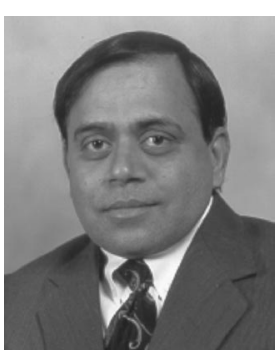

Ram Narayanan received his BTech degree in electrical engineering (electronics) from the Indian Institute of Technology, Madras (India), in 1976, and his PhD degree in electrical and computer engineering from the University of Massachusetts, Amherst, in 1988 . He is currently a professor of electrical engineering at the University of Nebraska-Lincoln. He has been working in the area of radar and laser remote sensing for over 15 years, and is primarily interested in instrument and algorithm development. Among his accom- plishments are the development of high-resolution imaging using random-noise radar, the development of midinfrared laser remotesensing technology, and the characterization of information content in remote-sensed images. He is currently establishing an Airborne Remote Sensing Facility at the University of Nebraska with funding from the National Science Foundation. He has published over 30 papers in refereed journals, and presented over 100 conference papers. $\mathrm{He}$ is active in the IEEE Geoscience and Remote Sensing Society. 